\title{
Properties of the radio jet emission of four gamma-ray Narrow Line Seyfert 1 galaxies
}

\author{
E. Angelakis*, L. Fuhrmann, I. Myserlis, I. Nestoras, V. Karamanavis, \\ T. P. Krichbaum, J. A. Zensus \\ Max-Planck-Institut für Radioastronomie, Auf dem Hügel 69, Bonn 53121, DE \\ E-mail: eangelakis@mpifr.de
}

\section{N. Marchili}

Dipartimento di Fisica ed Astronomia, Universita di Padova, via Marzolo 8, Padova 35131, IT

\section{Foschini}

Istituto Nazionale di Astrofisica, Osservatorio Astronomico di Brera, Via E. Bianchi, 46 23807,

Merate, IT

\section{H. Ungerechts, A. Sievers}

Instituto de Radio Astronomia Milimetrica, Avenida Divina Pastora 7, Local 20, E 18012

Granada, ES

\begin{abstract}
The detection of $\gamma$ rays from a small number of Narrow Line Seyfert 1 galaxies by the LAT instrument onboard Fermi seriously challenged our understanding of AGN physics. Among the most important findings associated with their discovery has been the realisation that smaller-mass black holes seem to be hosted by these systems. Immediately after their discovery a radio multifrequency monitoring campaign was initiated to understand their jet radio emission. Here the first results of the campaign are presented. The light curves and some first variability analyses are discussed, showing that the brightness temperatures and Doppler factors are moderate. The phenomenologies are typically blazar-like. The frequency domain on the other hand indicates intense spectral evolution and the variability patterns indicate mechanisms similar to those acting in the jets of BL Lacs and FSRQs. Finally, the linear polarisation also reveals the presence of a quiescent, optically thin jet in certain cases.
\end{abstract}

Nuclei of Seyfert Galaxies and QSOs - Central Engine and Conditions of Star Formation, November 6-8, 2012

Max-Planck-Insitut für Radioastronomie (MPIfR), Bonn, Germany

\footnotetext{
* Speaker.
} 


\section{Introduction}

The $\gamma$-ray emitting classes of Active Galactic Nuclei (hereafter AGN) that were thought to be there until 2008 were blazars - collectively referring to BL Lac objects (hereafter BL Lacs) and flat spectrum radio quasars (hereafter FSRQs) - and radio galaxies. In 2009 the LAT instrument onboard the Fermi-GST satellite detected high-energy $\gamma$ rays $(E>100 \mathrm{MeV})$ from the Narrow Line Seyfert 1 galaxy (NLSy1) PMN J0948+0022 with a blazar-like SED (Abdo et al. 2009a). The multi-wavelength campaigns that followed the discovery (Abdo et al. 2009b,a; Giroletti et al. 2011; Foschini et al. 2012) and the study of subsequently discovered NLSy1s (Abdo et al. 2009c) showed a clear blazar-like behaviour, indicating the existence of a relativistic jet viewed at small angles.

These detections have serious impact on our understanding of AGN. They challenge the belief that powerful relativistic jets form in giant elliptical galaxies since Seyferts mostly reside in disc galaxies (Sikora et al. 2007). Furthermore, they have implications on the energy production and dissipation at different scales because despite the fundamental differences between radio loud NLSy1s and blazars - in terms of black hole masses $\left(10^{6}-10^{8} \mathrm{M}_{\odot}\right)$ and accretion disc luminosities $\left(0.2-0.9 L_{\text {Edd }}\right)$, their jets seem to be behaving similarly and share the same properties. Hence they provide an excellent probe for studying the physics scaling towards lower black hole masses (see review by Komossa et al. 2006).

Here first results of a "single-dish" multi-frequency monitoring are reviewed and compared with those seen in typical AGNs. Focus is being put on the variability brightness temperatures as estimates of the energetics of flaring events and the variability pattern followed by the broad-band radio spectra as indicator of the variability and emission mechanism at play. The data discussed here are already partly published (Foschini et al. 2012) and partly in preparation for publication. Early results of this study are discussed by Fuhrmann et al. (2011).

\section{Light curves and variability}

Figure 1 shows the light curves along with the radio SEDs of the four NLSy1s that have been monitored with Effelsberg and IRAM telescopes. The monitoring program covers 2.6, 4.85, 8.35, 10.45, 14.60, 23.05, 32 and $43 \mathrm{GHz}$ with the Effelsberg 100-m telescope. For the two cases of J0324+3410 and J0948+0022 detectable with the IRAM 30-m telescope, also data at 86 and $142 \mathrm{GHz}$ are available. The mean cadence of the light curves and spectra shown there is approximately 1.5 months. Table 1 reports some indicative light curve parameters.

Apart from the case of $\mathrm{J} 0849+5108$ for which the dataset does not cover a substantial number of activity cycles, the remaining three sources show intense variability at practically all frequencies, a behaviour systematically seen in blazars. The variability amplitude is frequency dependent, following the typical fashion: higher frequencies vary more, which comprises yet another indication that variability mechanisms common with those acting in typical blazars, are present.

The phenomenologies seen in the light curves of these sources vary significantly and chiefly in terms of the observed power of the different events. In these terms J1505+0326 shows very weak flaring events. J0324+3410 shows at least two major events present at the high-end of the bandpass which disappear at lower frequencies. J0948+0022 show frequent events of activity which happen considerably fast. 

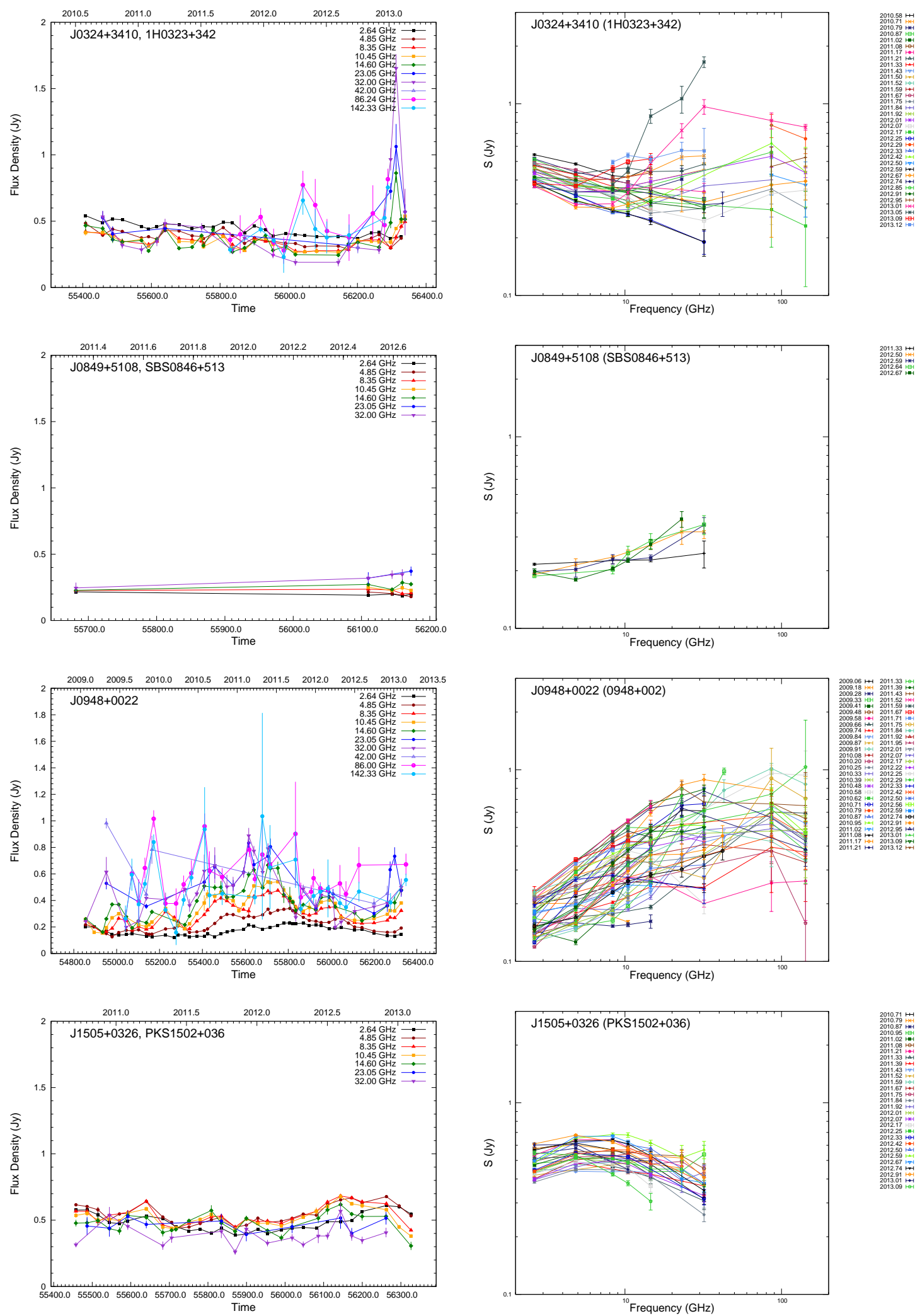

Figure 1: Multi-frequency light curves and radio SEDs of the four monitored NLSy1 galaxies. Each row corresponds to one source identified by its name in the top-left of the corresponding panel. The data points are connected with straight segments only for assistance to the eye. The axes limits are kept identical to allow inter-source comparisons. 
Table 1: Mean flux density and corresponding StDev for each observing frequency and source.

\begin{tabular}{llcccccccccc}
\hline \hline Source & $v^{1}$ & 2.64 & 4.85 & 8.35 & 10.45 & 14.6 & 23.05 & 32 & 43 & 86 & 142 \\
\hline \multirow{J}{*}{$0324+3410$} & $<\mathrm{S}>^{2}$ & 0.438 & 0.373 & 0.354 & 0.354 & 0.376 & 0.579 & 0.458 & 0.302 & 0.496 & 0.446 \\
& StDev $^{3}$ & 0.048 & 0.048 & 0.057 & 0.067 & 0.124 & 0.250 & 0.360 & - & 0.170 & 0.161 \\
\multirow{2}{*}{$0849+5108$} & $<\mathrm{S}>$ & 0.198 & 0.199 & 0.220 & 0.238 & 0.259 & 0.346 & 0.315 & - & - & - \\
& StDev & 0.011 & 0.018 & 0.015 & 0.012 & 0.026 & 0.037 & 0.048 & - & - & - \\
\multirow{2}{*}{$0948+0022$} & $<\mathrm{S}>$ & 0.171 & 0.224 & 0.301 & 0.336 & 0.390 & 0.551 & 0.469 & 0.608 & 0.590 & 0.502 \\
& StDev & 0.035 & 0.061 & 0.089 & 0.104 & 0.128 & 0.159 & 0.182 & 0.262 & 0.183 & 0.204 \\
J1505+0326 & $<$ S $>$ & 0.482 & 0.546 & 0.538 & 0.516 & 0.479 & 0.466 & 0.386 & - & - & - \\
& StDev & 0.066 & 0.070 & 0.075 & 0.067 & 0.071 & 0.047 & 0.080 & - & - & - \\
\hline
\end{tabular}

${ }^{1}$ Frequency in GHz.

${ }^{2}$ Mean flux density in Jy.

${ }^{3}$ Standard Deviation of around the mean flux density in Jy: measure of the variability amplitude.

\subsection{Variability brightness temperatures and Doppler factors}

Under the condition of long enough datasets, the previously discussed light curves can provide some insights into the energetics of the emitting regions responsible for the outbursts. From the observed variability amplitude and the time over which the event spans, one can work out the brightness temperature associated with the event, $T_{\mathrm{B}}$. In table 2 are reported the calculated maximum brightness temperatures at two characteristic frequencies, namely 8.35 (low band) and $32 \mathrm{GHz}$ (high band). Imposing an equipartition brightness temperature upper limit of $5 \cdot 10^{10} \mathrm{~K}$ (Readhead 1994), one can estimate the Doppler factors required to explain the observed excess. The levels of $T_{\mathrm{B}}$ seen in the three studied NLSy1s are rather moderate - compared to those usually seen in blazars - and closer to those of the lower luminosity blazars, namely the BL Lacs. Consequently, even at the high frequencies the inferred Doppler factors are moderate.

Table 2: Variability brightness temperatures and Doppler factors.

\begin{tabular}{|c|c|c|c|c|c|c|c|c|}
\hline \multirow[t]{2}{*}{ Source } & \multicolumn{4}{|c|}{$8.35 \mathrm{GHz}$} & \multicolumn{4}{|c|}{$32 \mathrm{GHz}$} \\
\hline & $\begin{array}{c}\Delta S \\
(\mathrm{Jy})\end{array}$ & $\begin{array}{l}\Delta t \\
\text { (d) }\end{array}$ & $\begin{array}{c}T_{\mathrm{B}, \max } \\
(\mathrm{K})\end{array}$ & $D$ & $\begin{array}{c}\Delta S \\
(\mathrm{Jy})\end{array}$ & $\begin{array}{l}\Delta t \\
(\mathrm{~d})\end{array}$ & $\begin{array}{c}T_{\mathrm{B}, \max } \\
(\mathrm{K})\end{array}$ & $\bar{D}$ \\
\hline $\mathrm{J} 0324+3410$ & 0.06 & 180 & $6 \cdot 10^{10}$ & 1 & 0.5 & 150.0 & $5 \cdot 10^{10}$ & 1 \\
\hline $\mathrm{J} 0948+0022$ & 0.07 & 150 & $4 \cdot 10^{12}$ & 5 & 0.2 & 320.0 & $2 \cdot 10^{11}$ & 2 \\
\hline $\mathrm{J} 1505+0326$ & 0.08 & 180 & $2 \cdot 10^{12}$ & 4 & 0.09 & 120.0 & $3 \cdot 10^{11}$ & 2 \\
\hline
\end{tabular}

\section{Broad-band spectra variability}

Figure 1 shows the time sampled broad-band spectra. The combined Effelsberg-IRAM spectra are simultaneous within 1.1 days securing that they are free of source variability noise. The mean sampling is one spectrum every 1.5 months (see also Sect. 2).

Except for J1505+0326 where the spectral evolution is not discernible, all other three NLSy1 would be classified as type " 1 " or " 2 " in the classification proposed by Angelakis et al. (2012) showing intense spectral evolution.

Despite the limited dataset, J0849+5108 behaves qualitatively similarly to J0948+0022. Both show very hard spectra with practically a complete absence of signs of a quiescence spectrum. The 


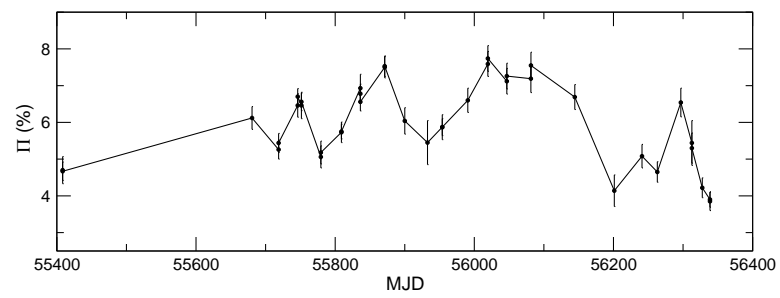

Figure 2: The fractional linear polarisation at $8.35 \mathrm{GHz}$ for $\mathrm{J} 0324+3410$. The effects of instrumental polarisation are, to first order, ignored but only significant detections (SNR better than 3) of polarised flux have been used.

spectra are highly inverted and intensely variable. In the case of J0948+0022 the source shows an impressive spectral evolution and remains at the hard-spectrum state (i.e. $\alpha \geq 0.0$ with $S \sim v^{\alpha}$ ) $4 \%$ of the time and its spectral index can reach values of +1.0 ; an indication of high frequency components dominating the observed emission. J0849+5108, on the other hand, shows flat spectra with a spectral index not exceeding values of the order of +0.2 .

In the case of J0324+3410 a steep spectrum component emerges at frequencies below 10.45 GHz. It appears intensely variable with the variability events transversing the bandpass, leaving however a "naked" steep spectrum behind. The low-band spectral index spends less than $3 \%$ of the time in negative values and can get as steep as -0.4 , very close to the commonly assumed values of -0.5 or -0.7 ; yet an indication of a large scale jet. The significant linear polarisation seen in the source also supports the idea of a significant contribution from a jet as it is discussed in Sect. 4.

J1505+0326 however imitates the behaviour of type " $5 \mathrm{~b}$ " in Angelakis et al. (2012) with only mild spectral evolution. Its spectrum is mostly convex, and at times shows high frequency components expanding fast towards lower bands.

\section{Linear polarisation}

Assuming that the variability seen in all the light curves discussed here is attributed to synchrotron self-absorbed components it is expected that significant linear polarisation is detected during their optically thin phase. The degree of polarisation is a function of the spectral index. Assuming highly ordered and intense magnetic fields (Sciama \& Rees 1967), as those expected during flares, even circular polarisation could occur. The superposition however of different components will result in "beam depolarisation".

Table 3 summarises the mean fractional linear polarisation $\langle\Pi\rangle$ and its standard deviation StDev for the four NLSy1s discussed here at $8.35 \mathrm{GHz}$. As it can be seen there, all four sources display detectable polarisation, though of relatively low values. J0324 +3410 however shows values larger than those typically observed in AGN at these frequency bands ( $\sim 4 \%$ at $5 \mathrm{GHz}$, e.g. Klein et al. 2003). Figure 2 shows its fractional linear polarisation light curve at $8.35 \mathrm{GHz}$. Its mean is of the order of $6 \%$ while at $4.85 \mathrm{GHz}$ it reaches values of $6.9 \%$. This phenomenology could be explained by assuming that the optically thin emission dominates the high frequency component (HFC) towards lower frequencies. The 4.85-GHz emission is less "contaminated" by the optically thick emission introduced by the HFC. It remains optically thin hence contributing higher fractional polarisation. Similar arguments hold for J0849+5108 although the effect is much more moderate. 
Table 3: Mean fractional linear polarisation at $8.35 \mathrm{GHz}$ of the four studied NLSy1s.

\begin{tabular}{cccccc}
\hline \hline Source & & $\mathrm{J} 0324+3410$ & $\mathrm{~J} 0849+5108$ & $\mathrm{~J} 0948+0022$ & $\mathrm{~J} 1505+0326$ \\
\hline$<\Pi>$ & $(\%)$ & 6.0 & 2.7 & 1.5 & 1.3 \\
StDev & $(\%)$ & 1.1 & 0.6 & 0.5 & 0.5 \\
\hline
\end{tabular}

Unlike J0324+3410, the other NLSy1s show very low polarisation degree. Their spectral variability pattern indicates that the sources undergo flaring events which "show" their optically thick part at the investigated frequency of $8.35 \mathrm{GHz}$ which is of low "emitted" polarisation. Polarisation measurements above $43 \mathrm{GHz}$ and above $10.45 \mathrm{GHz}$ for J0948+0022 and J1505+0326, respectively, should disclose a significant degree of polarisation.

\section{Discussion and conclusions}

This contribution is meant as a summary of the first results of the radio monitoring of four $\gamma$ loud NLSy1s. As expected, the sources show typical blazar-like behaviour with variable emission at practically all frequencies as already discussed by Fuhrmann et al. (2011). Outbursts causing changes in the flux density with factors up to 5 are seen. The variability brightness temperatures however do not dramatically exceed the theoretically imposed upper limits (equipartition). They imply rather moderate equipartition Doppler factors possibly associated with the systematically smaller black hole masses seen in these systems (Komossa et al. 2006). In the frequency domain on the other hand, the sources show very intense variability. Except for J1505+0326 which displays moderate evolution, the rest would be classified as type 1 or 2 according to Angelakis et al. (2012). The variability pattern indicates variability mechanisms similar to those acting in the jets of blazars. Specifically for J0948+0022 the evolution happens remarkably fast making it an excellent laboratory for the study of the spectral evolution of the high frequency component. The emergence of a steep spectrum component towards low bands for J0324+3410 and J0849+5108 and the increase of linear polarisation is yet another indication for the presence of an optically thin quiescent jet similar to that seen in typical blazars. A careful cross-correlation of the spectral index light curves with the fractional polarisation is underway to investigate the exact connection between the two and compare it with the synchrotron theory.

\section{References}

Abdo, A. A., Ackermann, M., Ajello, M., et al. 2009a, ApJ, 699, 976

Abdo, A. A., Ackermann, M., Ajello, M., et al. 2009b, ApJ, 707, 727

Abdo, A. A., Ackermann, M., Ajello, M., et al. 2009c, ApJ, 707, L142

Angelakis, E., Fuhrmann, L., Nestoras, I., et al. 2012, Journal of Physics Conference Series, 372, 012007

Foschini, L., Angelakis, E., Fuhrmann, L., et al. 2012, A\&A, 548, A106
Fuhrmann, L., Angelakis, E., Nestoras, I., et al. 2011, in Narrow-Line Seyfert 1 Galaxies and their Place in the Universe

Giroletti, M., Paragi, Z., Bignall, H., et al. 2011, A\&A, 528, L11

Klein, U., Mack, K.-H., Gregorini, L., \& Vigotti, M. 2003, A\&A, 406, 579

Komossa, S., Voges, W., Xu, D., et al. 2006, AJ, 132, 531

Readhead, A. C. S. 1994, ApJ, 426, 51

Sciama, D. W. \& Rees, M. J. 1967, Nature, 216, 147

Sikora, M., Stawarz, Ł., \& Lasota, J.-P. 2007, ApJ, 658, 815 